\title{
Vacunas en desarrollo: Helicobacter pylori
}

\author{
Paul Harris D., Carolina Serrano H. y Alejandro Venegas E.
}

\section{Vaccines under development: Helicobacter pylori}

Helicobacter pylori causes gastrointestinal disease including peptic ulcer and gastric cancer. An $H$. pylori vaccine is relevant because of the high prevalence of the infection and its associated complications. Extensive use of traditional antimicrobial therapies to eradicate $H$. pylori is not feasable, specially in developing countries, in part because of their high cost, associated adverse effects, the risk of reinfection, and the emergence of antimicrobial drug resistance. Numerous animal studies have been performed to determine infection outcomes and to explore the feasibility of a vaccine eradication or prevention of infection. These animal models with the possible exception of monkeys, have not been sufficient to address fundamental issues due to controversial results. A human model of $H$. pylori infection needs to be developed aimed to select an optimum vaccine candidate. The ultimate scientific goal will be to develop field studies using advanced vaccine candidates, but the current state of knowledge does is insufficient and has provided such candidates. These studies need to be designed in order to provide relevant information on immunity and pathogenesis associated to $H$. pylori.

Key words: vaccine, Helicobacter pylori, gastric cancer prevention.

Palabras clave: vacuna, Helicobacter pylori, prevención cáncer gástrico.

\section{Introducción}

$H$ elicobacter pylori es una bacteria gramnegativa que adquiere forma de $\mathrm{S}$, en $\mathrm{U}$, espiral o bacilar, microaerofílica y que cuenta con cuatro a siete flagelos polares. Es de crecimiento lento sobre medios suplementados con sangre o suero a $37{ }^{\circ} \mathrm{C}$ y se caracteriza bioquímicamente por la presencia de ureasa, catalasa y oxidasa. Su hábitat es el antro gástrico de los seres humanos y otros primates, donde se distinguen dos poblaciones bacterianas, una no-adherente, envejecida y no replicante $(98 \%)$ y una población adherente en activa replicación (2\%). La bacteria es capaz de interactuar con células epiteliales pero no invade los tejidos. La transmisión de la infección es de persona a persona y por mecanismos que son aún motivo de controversia (gastro-oral, oral-oral y fecaloral).

\section{¿Por que una vacuna contra Helicobacter pylori?}

- H. pylori infecta al 50\% de la población mundial, alcanzando tasas de infección hasta $90 \%$ en países en vías de desarrollo y $20 \%$ en países desarrollados. La prevalencia aumenta con la edad y con los indicadores de privación socioeconómica. A partir del Censo Nacional de Salud realizado en Chile en el año 2003, un grupo de investigadores de la Universidad Católica ha estudiado la prevalencia de $H$. pylori en esta población ${ }^{1}$ logrando determinar la concentración de anticuerpos de la clase IgG antiH. pylori $(\mathrm{AU} / \mathrm{ml})$ y establecer una prevalencia de la infección en población chilena adulta de $72,9 \%$ (IC 95\%: $70,0-75,9 \%)$.

- La infección por H. pylori es una causa importante de morbi-mortalidad, provocando patologías de consideración en $20 \%$ de los infectados. La mayoría de los infectados desarrolla una gastritis superficial crónica asintomática $(80 \%)$. Por razones no bien comprendidas, aproximadamente $15 \%$ de los infectados desarrollará úlcera gastroduodenal. Un número menor de ellos desarrollará una enfermedad linfo-proliferativa asociada al tejido linfoide de las mucosas (MALT) y otro grupo de infectados desarrollará una gastritis crónica atrófica, lesión preneoplásica capaz de desencadenar un adenocarcinoma gástrico $(0,1 \%)$. De hecho, $H$. pylori es considerado un carcinógeno tipo I desde 1994. Estudios epidemiológicos han demostrado la relación entre una serología positiva para $H$. pylori y el riesgo de adquirir un cáncer gástrico ${ }^{2-4}$. Dicha relación es probablemente temporal y progresiva, en la cual H. pylori es el primer evento y el cáncer gástrico el evento final. Entre ambos extremos sucede
Pontificia Universidad Católica de Chile

Facultad de Medicina Departamento de Pediatría, Sección de Gastroenterología (PHD, CSH) Facultad de Ciencias Biológicas Departamento de Genética Molecular y Microbiología (AVE)

Financiamiento parcial: Fondecyt 1030401 y 1030894 , Fondef D02I1067 y NIH DK54495.

Recibido: 8 agosto 2005 Aceptado: 10 octubre 2006

Correspondencia a:

Paul Harris Diez pharris@med.puc.cl 
una serie de situaciones agravantes o atenuantes, destacando factores genéticos y ambientales (polimorfismos de citoquinas como IL-1 $\beta$, factores de la dieta, vitamina $\mathrm{C}$, nitrosaminas, autoinmunidad, sales, secreción ácida, presencia de otras bacterias). Si bien la secuencia temporal clásica (infección $\rightarrow$ gastritis $\rightarrow$ atrofia $\rightarrow$ metaplasia $\rightarrow$ displasia $\rightarrow$ cáncer) ha sido puesta en duda, es claro que el elemento inicial es necesariamente la presencia de H. pylori.

- Problemas del tratamiento antimicrobiano. La erradicación de la bacteria con antibacterianos requiere de la asociación de múltiples fármacos ${ }^{5}$, lo que se asocia a mal cumplimiento de la terapia por parte del paciente y desarrollo de resistencia antimicrobiana por parte del patógeno ${ }^{6}$. Adicionalmente, una eficacia inconsistente, fármacos que no son accesibles en la mayoría de la población, costos para su uso en gran escala ${ }^{7}$, efectos adversos y el riesgo de reinfección, hacen recomendable el desarrollo de una vacuna.

\section{Factibilidad de una vacuna: Modelos animales}

Numerosos modelos animales que incluyen hurones, ratones, cerdos gnotobióticos y monos, han sido utilizados durante años para determinar el curso de la infección por $H$. pylori y la factibilidad de una vacuna ${ }^{8}$. El primer modelo disponible estuvo basado en $H$. felis ${ }^{9}$, lo que generó múltiples estudios en ratones con sonicados de $H$. felis en combinación con la toxina colérica (TC) como adyuvante, resultando en $96 \%$ de protección contra la infección subsecuente por $H$. felis ${ }^{10}$. Años de trabajo permitieron identificar cepas de $H$. pylori que infectaban exitosamente al ratón ${ }^{11,12}$, evolucionando hasta establecerse la cepa Sydney SS1 ${ }^{13}$. En la Tabla 1 se resumen estudios relevantes publicados en el modelo de ratón. Lisados o sonicados de $H$. pylori, en conjunción con adyuvantes como TC, fueron capaces de provocar disminuciones significativas en la carga bacteriana ${ }^{14}$. Algunos grupos reportaron un efecto significativo en dicha reducción, asociado a la ruta de vacunación y al adyuvante empleado, siendo utilizada preferentemente la aplicación intranasal de los antígenos crudos $^{15,16}$. La reciente utilización de los oligodesoxirribonucleótidos $\mathrm{CpG}$ como adyuvantes para evitar el efecto tóxico asociado a TC en humanos, ha sido evaluada por numerosos grupos observándose reducción significativa en la carga bacteriana ${ }^{16,17}$. Con respecto al mecanismo de dicha protección, Rahn y cols ${ }^{18}$ describieron un patrón diferencial de expresión proteica para ratones inmunizados versus infecta- dos, caracterizándose los primeros en un aumento de IFN- $\gamma$ y una serie de genes asociados a la expresión de dicha citoquina ${ }^{18}$. Por otra parte, estudios realizados por distintos grupos indican que ratones knock out (KO) para el gen de IFN- $\gamma$ aún presentan protección al ser inmunizados ${ }^{15,17,18}$. Garhart y cols ${ }^{15}$ describieron una disminución significativa en la protección observada al inmunizar en ratones KO para IL-12 y una correlación positiva entre la disminución de la carga bacteriana y la presencia de sintetasa de óxido nítrico inducible (iNOS), aún cuando la protección asociada a la inmunización se mantuvo en ratones KO. Garhart reportó también una disminución significativa en la carga bacteriana luego de inmunizar con antígeno crudo y desafiar con la cepa SS1 en ratones KO para IL-4, IL-5 y la producción de anticuerpos ${ }^{15,19}$. Dicha disminución fue dependiente del background genético del ratón a inmunizar; en ratones $\mathrm{BALB} / \mathrm{c}$ la ausencia de IL-12 da cuenta de una excesiva colonización en ratones infectados en contraposición al wild type (WT), observándose una reducción significativa de la carga bacteriana en protocolos de inmunización profiláctica ${ }^{20}$. La expresión de genes asociados a la vía Th1 se ve aumentada en los casos de inmunizaciones aunque los ratones $\mathrm{KO}$ para dichos genes sean capaces de mantener la protección, lo que lleva a pensar que son sistemas redundantes que permiten alcanzar la disminución de la carga bacteriana post inmunización.

En protocolos de vacunación terapéutica los resultados son bastante menos concluyentes. En ratones $\mathrm{BALB} / \mathrm{c}$, Kim y cols ${ }^{21}$ reportaron reducción significativa de la carga bacteriana asociada a la inmunización con lisados de SS1 más TC, reducción no observable en ratones C57BL/6. Por otro lado, Ravhagan y cols ${ }^{22}$ observaron con protocolos de inmunización terapéutica similares, una reducción significativa de la carga bacteriana al utilizar ratones con el mismo background $(\mathrm{C} 57 \mathrm{BL} / 6)^{22}$, sugiriendo que es indispensable que la cepa vacuna tenga el mismo antígeno de Lewis en su LPS que la cepa empleada en el desafío, para lograr algún efecto protector ${ }^{23}$.

La utilización de cepas atenuadas de Salmonella que expresan antígenos a elección es una alternativa a los protocolos de vacunación con lisados totales. Koesling y cols ${ }^{24}$ observaron una reducción significativa de la carga bacteriana al inmunizar profilácticamente con una dosis única de Salmonella enterica serovar Typhimurium con un sistema de expresión constitutivo de las subunidades A y B de la ureasa (UreAB) en ratones $\mathrm{C} 57 \mathrm{BL} / 6$ y $\mathrm{BALB} / \mathrm{c}$. En modalidad terapéutica sólo se observa reducción de la carga en ratones C57BL/ $6{ }^{24}$. Por otro lado, Londoño y cols ${ }^{25}$ obtuvieron resultados similares en protección y aumento de la respuesta humoral y celular, caracterizada esta última por un 
Tabla 1. Resumen de estudios de inmunizaciones con Helicobacter pylori en ratones

\begin{tabular}{|c|c|c|c|c|c|c|c|}
\hline Antígeno $^{a}$ & Ratón & Tipo $^{b}$ & $\begin{array}{l}\text { Adyuvante } \% \\
\text { carrier }\end{array}$ & $\begin{array}{c}\text { Vía de } \\
\text { administración }\end{array}$ & $\begin{array}{l}\text { Proteccióne } \\
(\%)\end{array}$ & $\begin{array}{l}\text { Seroconversión } \\
\text { Respuesta immune }\end{array}$ & Ref. \\
\hline Lisado SS1 & $\mathrm{C} 57 \mathrm{BL} / 6$ & $P$ & $\mathrm{CT}$ & IN & RSCB & 个IgG anti-H. pylori & 14 \\
\hline Lisado SS1 & C57BL/6 & $P$ & CT & OG & RSCB WT,IL4, p55, p55/75 KO & ND & 20 \\
\hline Lisado SS1 & $\mathrm{BALB} / \mathrm{C}$ & $P$ & CT & OG & RSCBWT,IL-4, IL-12 KO & ND & 20 \\
\hline Lisado SS1 & C57BL/6 & $P$ & CT & INIG & $\begin{array}{l}\text { RSCB solo IN en WT, IFN- } \gamma \text {, } \\
\text { iNOS KO, IL-12 KO } \sin R S C B\end{array}$ & 个IL-12 p40; IFN- $\gamma$, iNOS & 15 \\
\hline Lisado SS1 & C57BL/6 & $P$ & CT & IN & RSCB WT,IL-4, IL-5 KO & ND & 19 \\
\hline Lisado SS1 & $\mathrm{BALB} / \mathrm{CC} 57 \mathrm{BL} / 6$ & T & CT & OG & RSCB sólo BALB/C & 个IgG e IgA sérica y secretoria & 21 \\
\hline Lisado SS1 & C57BL/6 & T & CT & IG & $\mathrm{RSCB}$ & $\uparrow \lg G 2 \mathrm{a}$ & 22 \\
\hline $\begin{array}{l}\text { Lisado SS1 } \\
\text { Ley Hel } 305 \text { Le }^{x}\end{array}$ & C57BL/6 & T & CT & OG & $\begin{array}{c}\text { RSCB sólo cepa vacuna }= \\
\text { desafío }\end{array}$ & ND & 23 \\
\hline Lisado SS1 & $\mathrm{C} 57 \mathrm{BL} / 6$ & $P$ & $\mathrm{CpG}$ & SC & RSCB & $\uparrow$ IFN- $\gamma$, FNT- $\alpha$ & 17 \\
\hline Lisado SS1 & C57BL/6 & $P$ & СT & IN & RSCB & 个IFN- $\gamma$ genes asociados & 18 \\
\hline Lisado SS1 & $\mathrm{C} 57 \mathrm{BL} / 6$ & $P$ & $\mathrm{CpG}$ & IN & RSCB & $\begin{array}{c}\text { IFN- } \gamma \text { KO cae protección } \\
\text { e inflamación }\end{array}$ & 16 \\
\hline UreAB constitutivo & C57BL/6 & $\mathrm{P} / \mathrm{T}$ & /S. Typhimurium aroA & 0 & RSCB & $\uparrow \lg G 2 \mathrm{a}$ & 24 \\
\hline UreAB constitutivo & $\mathrm{BALB} / \mathrm{C}$ & $\mathrm{P} / \mathrm{T}$ & $\begin{array}{l}\text { /1 dosis } \\
\text { S. Typhimurium aroA }\end{array}$ & 0 & RSCB sólo profiláctico & ND & 24 \\
\hline UreAB inducible & $\mathrm{BALB} / \mathrm{C}$ & $P$ & IS. enterica serovar Typhi & $I N+B$ & RSCB luego del booster & IFN- $\gamma$, IL-10, IL-5 & 25 \\
\hline pcDNA hspA, hspB & $\mathrm{C} 57 \mathrm{BL} / 6$ & $P$ & /Plásmido & IC & RSCB sólo hspB & ND & 26 \\
\hline p-kat (catalasa) & $\mathrm{C} 57 \mathrm{BL} / 6$ & $P$ & /Plásmido & INIC & RSCB ambas vías & $\uparrow \lg G, \lg A$ & 27 \\
\hline UreB & C57BL/6 & $\mathrm{P} / \mathrm{T}$ & /poliovirus & $\mathrm{IM}$ & RSCB & $\begin{array}{l}\text {-IgG, Mod. IL-10, IL-4, IFN- } \gamma \\
\text { sólo profiláctica }\end{array}$ & 28 \\
\hline
\end{tabular}

a Ure $A B=$ subunidades $A$ y $B$ ureasa, Ure $B=$ subunidad $B$ ureasa, Kat $=$ catalasa, hsp $A=$ proteína de shock térmico $A$, hsp $B=$ proteína de shock térmico $B$, ${ }^{\mathrm{b} P}=$ profiláctica, $\mathrm{T}=$ terapéutica; ${ }^{\mathrm{C}} \mathrm{CT}=$ toxina colérica, $\mathrm{CpG}=$ oligodesoxirribonucleótido ${ }^{\mathrm{d}}$

$\mathrm{IN}=$ intranasal, $\mathrm{OG}=$ orogástrico, $\mathrm{IG}=$ intragástrico, $\mathrm{SC}=$ subcutáneo, $\mathrm{O}=$ oral, $\mathrm{IN}+\mathrm{B}=$ intranasal + booster, $\mathrm{IC}=$ intracutáneo, $\mathrm{IM}=$ intramuscular; e RSCB=Reducción significativa de la carga bacteriana; $\mathrm{WT}=$ wild type; ${ }^{\mathrm{N}} \mathrm{ND}=$ no determinado, $\mathrm{NC}=$ no corresponde, $\mathrm{KO}=\mathrm{Knock}$ out.

aumento inespecífico en la secreción de citoquinas, al utilizar S. enterica serovar Typhi con un sistema de expresión para UreAB inducible, en modalidad profiláctica. Dicha protección y aumento en la respuesta inmune estaría asociada a la inmunización primaria más la presencia de un booster luego de varias semanas $^{25}$.

La administración de vacunas de ADN utilizando antígenos como ureasa, catalasa y proteínas de shock térmico (heat shock protein-hsp) A y B, mostraron también reducción significativa de la carga bacteriana en administraciones intracutáneas e intranasales de los plásmidos vacuna ${ }^{26,27}$.

La búsqueda de modalidades de vacuna ha llevado al desarrollo de sistemas bastante novedosos como son vectores virales para la expresión de antígenos de H. pylori. Smythies reportó la utilización de poliovirus al cual se le han remplazado los genes de la cápside con UreB, tanto en modalidades profilácticas como terapéuticas de inmunización; con esta estrategia se observa reducción de la carga bacteriana y en el caso de vacunación profiláctica, modificación además de los patrones de secreción de citoquinas ${ }^{28}$.
En conjunto, estos estudios demuestran la factibilidad de la reducción en la carga bacteriana de manera significativa, aunque generalmente no es posible lograr la desaparición completa de $H$. pylori. Aún cuando se observan modificaciones en los patrones de secreción de citoquinas y un aumento en la respuesta humoral asociada a las inmunizaciones, todavía es controversial si estos resultados son reproducibles en seres humanos.

\section{Ensayos de vacunas en seres humanos}

Aún cuando varios antígenos de $H$. pylori protectores en ratones son capaces de gatillar respuestas humorales y celulares, no se ha podido identificar un marcador inmune de protección. Así los estudios de campo en humanos son la mejor alternativa para evaluar potenciales vacunas, utilizando el porcentaje de erradicación o protección contra $H$. pylori como variables a analizar. La Tabla 2 resume los estudios efectuados en humanos y publicados a la fecha de redactar este artículo. 


\begin{tabular}{|c|c|c|c|c|c|c|c|c|}
\hline Antígeno a & $\mathbf{N}$ & Tipo b & $\begin{array}{l}\text { Adjuvante } \\
\text { /carrier }\end{array}$ & $\begin{array}{c}\text { Vía de } \\
\text { administración }\end{array}$ & $\begin{array}{c}\text { Protección } \\
(\%)\end{array}$ & $\begin{array}{l}\text { Erradicación }^{\text {d }} \\
(\%)\end{array}$ & $\begin{array}{c}\text { Seroconversión } \\
\text { Respuesta immune }\end{array}$ & Ref. \\
\hline Lisado H. pylori & 23 & $\mathrm{~T} / \mathrm{P}$ & LT / - & Oral & ND & 0 & $50-70 \%$ & 29 \\
\hline Lisado H. pylori & 5 & $P$ & $\mathrm{LT} /$ - & Oral & ND & NC & $40 \%$ & 30 \\
\hline UreAB & 12 & $\mathrm{~T}$ & - & Oral & NC & 0 & NC & 31 \\
\hline UreAB & 26 & $\mathrm{~T}$ & $\mathrm{LT} /$ - & Oral & NC & RSCB & NC, aumento & 32 \\
\hline UreAB & 42 & $P$ & $\mathrm{LT} /$ - & Oral & ND & NC & $22 \%$ & 33 \\
\hline UreAB & 18 & $P$ & $\mathrm{LT} /$ - & Rectal & ND & NC & $25 \%$ & 34 \\
\hline UreAB & 8 & $P$ & $\begin{array}{c}\text { - / S. enterica serovar } \\
\text { Typhi Ty800 }\end{array}$ & Oral & ND & NC & $0 \%$ & 35 \\
\hline UreAB & 6 & $P$ & $\begin{array}{c}\text { - / S. enterica serovar } \\
\text { Typhimurium }\end{array}$ & Oral & ND & NC & $50 \%$ & 36 \\
\hline UreAB & 9 & $P$ & $\begin{array}{c}\text { - / S. enterica serovar } \\
\text { Typhi Ty21a }\end{array}$ & Oral & ND & NC & $25 \%$ & 37 \\
\hline UreAB & 13 & $P$ & $\begin{array}{c}\text { - / S. enterica serovar } \\
\text { Typhi Ty21a }\end{array}$ & Oral & ND & NC & $56 \%$ & 38 \\
\hline $\begin{array}{l}\text { CagA } \\
\text { VacA } \\
\text { NAP }\end{array}$ & $?$ & $P$ & Aluminio / - & IM & ND & NC & $100 \%$ & 39 \\
\hline 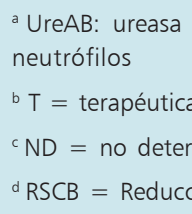 & $\begin{array}{l}\text { mbin } \\
=\text { pro } \\
\text { ado, } \\
\text { signif }\end{array}$ & $\begin{array}{l}\text { (subunid } \\
\text { tica } \\
=\text { no corr } \\
\text { va de la }\end{array}$ & $\begin{array}{l}\text { ades } A \text { y } B), \text { Cag } A= \\
\text { esponde, } \quad I M=\text { intra } \\
\text { arga bacteriana, }\end{array}$ & $\begin{array}{l}\text { ntígeno } A \text { asociado } \\
\text { muscular }\end{array}$ & xina, VacA & ina vacuolizant & NAP = proteína active & ora $d$ \\
\hline
\end{tabular}

Vacunas con bacterias inactivadas. Kotloff y $\operatorname{cols}^{29}$ estudiaron vacunas basadas en bacterias enteras inactivadas en formalina (Helivax $\left.{ }^{\circledR}\right)$. Se han realizado dos estudios fase I, en los cuales la vacuna es administrada vía oral usando como adyuvante la toxina termo lábil de Escherichia coli (LT). En el primer estudio, 23 sujetos adultos con o sin infección por $H$. pylori fueron estudiados en base a un esquema dosis-respuesta. En la segunda fase, 18 pacientes infectados, en forma randomizada recibieron placebo o vacuna, vía oral, en tres dosis. Si bien ninguno de ellos fue capaz de erradicar $H$. pylori, se observó un aumento en la secreción de IgA específica salival y fecal, tanto en infectados como no infectados, y un aumento en IFN$\gamma$ en $70 \%$ en los sujetos no infectados, así como también se observaron respuestas linfoproliferativas en los pacientes no infectados. La presencia de efectos adversos significativos como diarrea (26\%), fiebre $(22 \%)$ y vómitos $(9 \%)$ sugiere que LT posee numerosos efectos adversos ${ }^{29}$.

En un segundo estudio se administró Helivax ${ }^{\circledR}$ en conjunto con LT mutante menos tóxica (LTR 192G) a cinco adultos no infectados en base a un diseño de tres dosis. De ellos, dos voluntarios presentaron respuestas celulares y se demostró que esta respuesta era mayor en las biopsias duodenales que en las antrales, constituyéndose en la primera demostración en seres humanos de una respuesta inmune contra $H$. pylori de tipo B en mucosa ${ }^{30}$.

Vacunas con adyuvantes de mucosa. El grupo de Michetti y cols administraron Ure AB sin adyuvante en un diseño doble ciego, placebo controlado, randomizado y con una dosis semanal durante cuatro semanas a 12 adultos infectados y asintomáticos. Ninguno de los pacientes erradicó $H$. pylori y no tuvo diferencias histológicas, clínicas o bioquímicas antes y después del ensayo, lo que demostró que UreAB en ausencia de adyuvantes, no producía ningún cambio en el curso de la infección ${ }^{31}$.

En un segundo estudio de este grupo, se administró UreAB más LT a 26 adultos en un esquema similar al descrito previamente. No se pudo demostrar erradicación de $H$. pylori en ninguno de los pacientes, pero se observó el aumento de $\operatorname{IgA}$ anti ureasa con las dosis mayores de Ure AB y una disminución en la densidad de la bacteria en el estómago con las dosis 
más bajas. La alta tasa de diarrea observada sugiere que el uso de LT hace poco practicable el desarrollo de una vacuna en estas condiciones ${ }^{32}$.

En un tercer trabajo de los mismos autores, exploraron el uso de UreAB con LT en dosis inferiores (0-2,5 $\mu \mathrm{g})$ en 42 adultos no infectados con H. pylori. En este estudio doble ciego, placebo control, randomizado, los pacientes recibieron una dosis oral de $60 \mathrm{mg}$ de antígeno semanal, durante cuatro semanas. Se observó respuesta inmune ( $\operatorname{IgG}$ e $\operatorname{Ig} \mathrm{A}$ ) en $22 \%$ de los pacientes y una disminución en la densidad de $H$. pylori. Hubo cerca de $50 \%$ de efectos adversos atribuibles a $\mathrm{LT}^{33}$.

Finalmente, en un estudio reciente, el mismo grupo de investigadores entregó Ure $\mathrm{AB}$ por vía rectal con o sin adyuvante (LT $5-25 \mathrm{mg}$ ). Se estudiaron 18 sujetos asintomáticos no infectados en forma randomizada, doble ciego, con dosis ascendentes de UreAB, en tres dosis de vacuna repartidas a lo largo de un mes. Se observó una seroconversión a LT en 60 a $80 \%$ de los vacunados y 8 a $25 \%$ a ureasa ${ }^{34}$.

Vacunas con vectores vivos. La utilización de Salmonella Typhi modificada con una deleción de la región de virulencia phoP/phoQ ha demostrado ser segura e inmunogénica en dosis única y oral como vacuna contra fiebre tifoidea. En un primer estudio por Dipetrillo y cols ${ }^{35}, S$. Typhi fue modificada con la introducción de la UreAB (S. Typhi TY1033). En este estudio 7 sujetos no infectados recibieron una dosis única oral de TY1033 y un octavo voluntario recibió dos dosis tres meses más tarde. Los efectos adversos fueron similares a lo observado con el vector no modificado. Los 8 pacientes presentaron una fuerte respuesta inmune de mucosa dirigida contra antígenos del vector pero ninguno tuvo respuesta detectable a ureasa después de la inmunización. Un subgrupo de 3 voluntarios recibió un refuerzo oral consistente de UreAB más LT, 15 días después de la inmunización; ninguno tuvo una respuesta detectable a la ureasa ${ }^{35}$ Angelakopoulos y cols ${ }^{36}$ utilizaron una $S$. enterica serovar Typhimurium atenuada (delección phoP/phoQ) y modificada de la misma manera con UreAB. En este estudio, 6 sujetos no infectados fueron vacunados, observándose $50 \%$ de seroconversión en los sujetos que recibieron vacunación profiláctica. Cinco voluntarios presentaron una respuesta serológica al vector y 3 de ellos respuesta a UreAB. Sólo 2 voluntarios presentaron fiebre. La modesta mejoría en la respuesta inmune comparada con el ensayo anterior podría explicarse por diferencias en la colonización por el vector o mejoría en la expresión antigénica.

Otro estudio del grupo inicial utilizó el mismo antígeno UreAB en la cepa mutante de $S$. enterica serovar Typhi contenida en la vacuna Ty $21 \mathrm{a}^{37}$. Nueve sujetos no infectados recibieron la vacuna con antígenos y 3 voluntarios no infectados recibieron sólo el vector bacteriano. Ninguno de ellos presentó efectos adversos. Diez de 12 presentaron una respuesta inmune humoral contra Salmonella, pero sólo 2 voluntarios seroconvirtieron. Cinco de 12 presentaron respuesta en 1 o 2 de los 3 ensayos para respuesta celulares al carrier (proliferación, secreción de IFN- $\gamma$ ). Tres de 12 presentaron una débil pero significativa respuesta celular frente a la ureasa de $H$. pylori, mientras que ningún voluntario tuvo una respuesta humoral detectable a la ureasa. Finalmente, en un estudio reciente del año $2004^{38}, 13$ voluntarios no infectados fueron preinmunizados con la cepa vaccinal para posteriormente recibir un esquema de tres dosis de vacunas viva Ty21a más UreAB. Nueve pacientes recibieron cepa vacuna con los antígenos y 4 sujetos con la cepa vaccinal sin los antígenos. Nuevamente no se observaron efectos adversos y 5 de 9 pacientes que recibieron vacunas con antígenos presentaron respuesta inmune celular al antígeno.

Vacunas parenterales. Las proteínas purificadas son pobremente inmunogénicas por vía oral y no obstante, la mayoría de los estudios a la fecha han sido realizados por esta ruta y con un solo antígeno simultáneo. Los pobres resultados obtenidos con las metodologías previas han estimulado el desarrollo de vacunas parenterales basadas en la utilización de múltiples antígenos. Es así como en el año 2002 y 2003 se publicaron estudios clínicos en humanos para una vacuna intramuscular basada en tres antígenos (VacA, CagA, NAP) en dosis variable de 10 a $25 \mu \mathrm{g}$, empleando hidróxido de aluminio como adyuvante. En ellos se intentaron distintos esquemas de tres dosis cada uno con resultados positivos, comprobándose la presencia de IgG sérica contra los tres antígenos en todos los sujetos, respuesta inmune celular contra CagA y ausencia de efectos adversos, pero no se estudió la eficacia contra la colonización ${ }^{39}$.

\section{Nuevas estrategias}

Estudios más recientes del año 2003, sugieren que la iniciación de respuestas inmune vía mucosa con mutantes de LT como adyuvantes, ya sea vía oral o intranasal, pudiera ser una primera etapa para una posterior ruta parenteral con emulsión de aceite-agua como adyuvantes ${ }^{40}$. También se estudia activamente la mejor vía para inducir respuesta en humanos donde estudios preliminares sugieren que la ruta oral o intrayeyunal es claramente mejor que la rectal o na$\mathrm{sal}^{41}$.

El desarrollo de vacunas se ha enlentecido por la 
búsqueda de antígenos protectores. En este sentido, el análisis bio-informático y experimental de proteomas proporciona información global sobre propiedades antigénicas relevantes. Así se ha establecido una lista de factores de virulencia de los cuales varios se han identificado y analizado en ensayos preclínicos de inmunización. El análisis de proteomas permite evaluar propiedades como abundancia, localización y seroreactividad. Parece lógico, por tanto, combinar los tres criterios mencionados para seleccionar antígenos potenciales para vacunas ${ }^{42}$.

Sin embargo, el mayor desarrollo a futuro parece estar dado por la implementación de protocolos de infecciones experimentales con $\mathrm{H}$. pylori en humanos voluntarios ${ }^{43}$. El año 2004 aparecen publicados los primeros modelos de desafío de $H$. pylori en seres humanos lo cual ha permitido estudiar aspectos histológicos, fisiología de la secreción ácida, y que en un futuro cercano permitirán estudiar potenciales vacunas $^{44}$

\section{Conclusiones}

El desarrollo de una vacuna contra $H$. pylori continúa estando en un plano hipotético. La vacuna profiláctica basa sus fundamentos en que la infección se adquiere en la niñez, y que las secuelas son el resultado de la infección crónica. Al mismo tiempo, el desarrollo de una vacuna terapéutica cobra validez al considerar que $50 \%$ de la población mundial está infectada y que la utilización de una vacuna puede ser una alternativa frente a la falla de tratamiento y evitando la reinfección.

La ausencia de correlato inmunológico de protección sugiere con fuerza la necesidad de realizar estu- dios clínicos fase III. Muchos temas permanecen sin resolver, entre ellos la dificultad de lograr una completa erradicación en animales, la ausencia de adyuvantes seguros y potentes en seres humanos y la preocupación por el eventual riesgo de exacerbar patologías en pacientes ya infectados.

\section{Resumen}

La infección con Helicobacter pylori es la causa de patologías gastrointestinales como úlcera péptica y cáncer gástrico. Una vacuna contra $H$. pylori es relevante debido a la alta prevalencia de la infección y a la morbi-mortalidad asociada a ésta en nuestro país. El uso masivo de terapias antimicrobianas actuales no es una estrategia factible, especialmente en países en desarrollo, en parte debido al alto costo, los múltiples efectos adversos, el riesgo de reinfección y la emergencia de resistencia a los antimicrobianos. Numerosos modelos animales han sido utilizados durante años para determinar el curso de la infección por $H$. pylori y explorar la factibilidad de una vacuna, ya sea para erradicar o prevenir la infección. Dichos modelos, con la posible excepción de los monos, no son suficientes para responder preguntas fundamentales debido a resultados contradictorios. Un modelo humano de infección por H. pylori debe ser desarrollado con el principal propósito de seleccionar vacunas óptimas. El objetivo final es el desarrollo de estudios de campos de vacunas candidatas, pero el estado actual del conocimiento no proporciona una metódica adecuada para seleccionar tales vacunas candidatas promisorias. Dichos estudios pueden ser diseñados para proporcionar información relevante sobre la inmunidad y patogénesis de la infección por H. pylori.

\section{Referencias}

1.- Rollán A, Ferreccio C, Harris P, Serrano C, Jara A, Venegas A. Early Helicobacter pylori infection is related to gastric cancer risk in Chile, a high-risk area: A population-based study. Can J Gastroenterol 2005; 19-43.

2.- Nomura A, Stemmermann G N, Chyou P H, et al. Helicobacter pylori infection and gastric carcinoma among Japanese Americans in Hawaii. N Engl J Med 1991; 325: 1132-6.

3.- Parsonnet J, Blaser M J, Pérez-Pérez G I, et al. Symptoms and risk factors of Helicobacter pylori infection in a cohort of epidemiologist. Gastroenterol 1992; 102:
41-6.

4.- Forman D, Newell D G, Fullerton F, et al. Association between infection with Helicobacter pylori and risk of gastric cancer: evidence from a prospective investigation. Br Med J 1991; 302: 1302-5.

5.- Treiber G. The influence of drug dosage on Helicobacter pylori eradication: a costeffectiveness analysis. Am J Gastroenterol 1996; 9: 246-57.

6.- Megraud F. Basis for the management of drug-resistant Helicobacter pylori infection. Drugs 2004; 64: 1893-904.

7.- Ford A C, Delaney B C, Forman D, Moayyedi P. Eradication therapy in Helicobacter pylori positive peptic ulcer disease: systematic review and economic analysis. Am J Gastroenterol 2004; 99: 1833-55.

8.- Kusters J G. Recent developments in Helicobacter pylori vaccination. Scan J Gastroenterol 2001; 36 Suppl 234: 15-21.

9.- Lee A, Fox J G, Otto G, Murphy J A. Small animal model of human and Helicobacter pylori active chronic gastritis. Gastroenterol 1999; 99: 1315-23.

10.- Czinn S J, Nedrud J G. Protection of germfree mice from infection by Helicobacter felis after active oral or passive IgA immunization. Vaccine 1993; 11: 637-42.

11.- Cellini L, Allocati N, Angelucci D, Iezzi T, Di Campli E, Marzio L, et al. Coccoid 
Helicobacter pylori not culturable in vitro reverts in mice. Microbiol Immunol 1994; 38: $843-50$.

12.- Marchetti M, Arico B, Burroni D, Figura N, Rappuoli R, Ghiara P. Development of a mouse model of Helicobacter pylori infection that mimics human disease. Science 1995; 267: 1655-8.

13.- Lee A, O'Rourke J, De Ungria M C, Robertson B, Daskalopoulos G, Dixon M F. A standardized mouse model of Helicobacter pylori infection: introducing the Sydney strain. Gastroenterol 1997; 112: 1386-97.

14.- Garhart C A, Redline R W, Nedrud J G, Czin SJ. Clearance of Helicobacter pylori infection and resolution of postimmunization gastritis in a kinetic study of prophylactically immunized mice. Infect Immun 2002; 70: 3529-38.

15.- Garhart C A, Heinzel F P, Czin S J, Nedrud J G. Vaccine induced reduction of Helicobacter pylori colonization in mice is interleukin-12 dependent but gamma interferon and inducible nitric oxide synthase independent. Infect Immun 2003; 71: 910-21.

16.- Shi T, Liu W Z, Gao F, Shi G Y, Xiao S D. Intranasal CpG-oligodeoxynucleotide is a potent adjuvant of vaccine against Helicobacter pylori, and $\mathrm{T}$ helper I type response and IFN- $\gamma$ correlate with the protection. Helicobacter 2005; 10: 71-9.

17.- Sommer F, Wilken H, Faller G, Lohoff M. Systemic Th1 immunization of mice against Helicobacter pylori infection with $\mathrm{CpG}-$ oligodeoxynucleotides as adjuvants does not protect from infection but enhances gastritis. Infect Immun 2004; 72: 1029-35.

18.- Rahn W, Redline R W, Blanchard T G. Molecular analysis of Helicobacter pyloriassociated gastric inflammation in naïve versus previously immunized mice. Vaccine 2004; 23: 807-18

19.- Garhart C A, Nedrud J G, Heinzel F P, Sigmund N E, Czin S J. Vaccine induced protection against Helicobacter pylori in mice lacking both antibodies and interleukin4. Infect Immun 2003; 71: 3628-33.

20.- Panthel K, Faller G, Haas R. Colonization of C57BL/6J and BALB/c wild-type and knockout mice with Helicobacter pylori: Effect of vaccination and implications for innate and acquired immunity. Infect Immun 2003; 71: 794-800.

21.- Kim J S, Chang J H, Chung S, Yum J S. Importance of host genetic background on immune response to Helicobacter pylori infection and therapeutic vaccine efficacy.
FEMS Immun Med Microbiol 2001; 31: 416.

22.- Raghavan S, Svennerholm A M, Homgren J. Effects of oral vaccination and immunomodulation by cholera toxin on experimental Helicobacter pylori infection, reinfection, and gastritis. Infect Immun 2002; 70: 4621-7.

23.- Raghavan S, Hjustrom M, Homgren J Svennerholm A M. Protection against experimental Helicobacter pylori immunization with inactivated H. pylori whole-cell vaccines. Infect Immun 2002; 70: 6383-8.

24.- Koesling J, Lucas B, Develioglou L, Aebischer T, Meyer T F. Vaccination of mice with live recombinant Salmonella typhimurium aroA against $H$. pylori: parameters associated with prophylactic and therapeutic vaccine efficacy. Vaccine 2001; 20: 413-20.

25.- Londoño-Arcila $P$, Freeman $D$, Kleathous $H$, O'Dowd A M, Lewis S, Turner AK, et al. Attenuated Salmonella enterica serovar Typhi expressing urease effectively immunized mice against Helicobacter pylori challenge as part of a heterologous mucosal priming-parenteral boosting vaccination regimen. Infect Immun 2002; 70: 5096-106.

26.- Todoroki I, Joh T, Watanabe K, Miyashita M, Seno K, Nomura T, et al. Suppressive effects of DNA vaccines encoding heat shock proteins on Helicobacter pylori-induced gastritis in mice. Biochem Biophys Res Commun 2000; 277: 159-63.

27.- Miyashita M, Joh T, Watanabe K, Todoroki I, Seno K, Ohara H, et al. Immune response in mice to intranasal and intracutaneous administration of a DNA vaccine encoding Helicobacter pyloricatalase. Vaccine 2002; 20: 2336-42.

28.- Smythies L E, Novak M J, Waites K B, Lindsey J R, Morrow C D, Smith P D. Poliovirus replicons encoding the $\mathrm{B}$ subunits of Helicobacter pylori urease protect mice against $H$. pylori infection. Vaccine 2005; 23: 901-9.

29.- Kotloff K L, Sztein M B, Wasserman S S, Losonsky G A, DiLorenzo S C, Walker R I. Safety and immunogenicity of oral inactivated whole-cell Helicobacter pylori vaccine with adjuvant among volunteers with or without subclinical infection. Infect Immun 2001; 69: 3581-90.

30.- Losonsky G, Kotloff K, Walker R. B cell responses in gastric antrum and duodenum following oral inactivated Helicobacter pylori whole cell (HWC) vaccine and LT R192G in $H$. pylori seronegative individuals. Vaccine 2003; 21: 562-5.

31.- Kreiss C, Buclin T, Cosma M, CorthesyTheulaz I, Michetti P. Safety of oral immunisation with recombinant urease in patients with Helicobacter pylori infection. Lancet 1996; 347: 1630-1.

32.- Michetti P, Kreiss C, Kotloff K, et al. Oral immunization with urease and $E$. coli heatlabile enterotoxin is safe and immunogenic in Helicobacter pylori-infected adults. Gastroenterol 1999; 116: 804-12.

33.- Banerjee S, Medina-Fatimi A, Nichols R, Tendler D, Michetti M, Simon J, et al. Safety and efficacy of low dose Escherichia coli enterotoxin adjuvant for urease based oral immunisation against Helicobacter pylori in healthy volunteers. Gut 2002; 51 : 634-40.

34.- Sougioultzis S, Lee C K, Alsahli M, Banerjee S, Cadoz M, Schrader R, et al. Safety and efficacy of $E$. coli enterotoxin adjuvant for urease-based rectal immunization against Helicobacter pylori. Vaccine 2002; 21: 194-201.

35.- DiPetrillo M D, Tibbetts T, Kleanthous H, Killeen K, Hohmann E L. Safety and immunogenicity of phoO/phoQ-deleted Salmonella typhi expressing Helicobacter pylori urease in adult volunteers. Vaccine 1999; 18: 449-59.

36.- Angelakopoulos H, Hohmann E L. Pilot study of phoO/phoQ-deleted Salmonella enterica serovar Typhimurium expressing Helicobacter pylori urease in adult volunteers. Infect Immun 2000; 68: 2135-41.

37.- Bumann D, Metzger W, Mansouri E, Palme O, Wendland M, Hurwitz R, et al. Safety and immunogenicity of live recombinant Salmonella enterica serovar Typhi Ty21a expressing urease A and B from Helicobacter pylori in human volunteers. Vaccine 2001; 20: 854-52.

38.- Metzger W, Mansouri E, Kronawitter M, Diescher S, Soerensen M, Hurwitz R, et al. Impact of vector-priming on the immunogenicity of a live recombinant Salmonella enterica serovar Typhi Ty21a vaccine expressing urease A and B from Helicobacter pylori in human volunteers. Vaccine 2004; 22: 2273-7.

39. - Malfertheiner P, Schultze V, Del Giudice G, Rosenkranz B, Kaufmann S H E, Winau F, et al. Phase I safety and immunogenicity of a three-component $H$. pylori vaccine. Gastroenterol 2002, S 122, A-585. 
40.- Ruggiero P, Peppoloni S, Rappuoli R, Del Giudice $\mathrm{G}$. The quest for a vaccine against Helicobacter pylori: how to move from mouse to man? Microbes Infect 2003; 5: 749-56.

41.- Johansson E L, Bergquist C, Edebo A, Johansson C, Svennerholm A M. Comparison of different routes of vaccination for eliciting antibody responses in the human stomach. Vaccine 2004; 22: 984-90.

42.- Bumann D, Jungblut $P$, Meyer T. Helicobacter pylori vaccine development based on combined subproteome analysis. Proteomics 2004; 4: 2843-8.

43.- Michetti P. Experimental Helicobacter pylori infection in humans: a multifaceted challenge. Gut 2004; 53: 1220-1.

44.- Graham D Y, Opekun A R, Osato M S, El-Zimaity H M, Lee CL, Yamaoka Y, et al. Challenge model for Helicobacter pylori infection in human volunteers. Gut 2004; 53: $1235-43$. 